\title{
Carbon dots: New Biomaterials in Surface Science ${ }^{\dagger}$
}

\author{
Roger M. Leblanc ${ }^{1}$ \\ 1 Department of Chemistry, University of Miami, USA \\ * Correspondence: e rml@ miami.edu; \\ $\uparrow$ Presented at Materials Chemistry and Physics (Materials Chemistry 2020) - International e-Conference
}

Received: 16.09.2020; Revised: 20.09.2020; Accepted: 24.09.2020; Published: 27.09.2020

\begin{abstract}
Carbon dots (CDs) with size less than $10 \mathrm{~nm}$ have recently triggered great attention in the research of material science and biomedical engineering due to their unique properties such as small size, excellent photoluminescence (PL), high water-dispersity, biocompatibility, nontoxicity, and abundant surface functionalities. In this presentation, I will firstly introduce diverse preparations for CDs. Extensive structural characterizations have been used to hypothesize comprehensive structural models for 3 distinct CD species that represent both top-down and bottom-up approaches in order to optimize their properties and applications.

Then, I will mainly focus on many excellent biomedical applications of the CDs recently developed in our lab: (1), in vivo experiment suggested that glucose-based CDs could cross the blood-brain barrier (BBB) due to the presence of glucose transporter proteins on the BBB; (2), a drug delivery system of carbon nitride dots conjugated with an anti-cancer therapeutic drug and a targeting molecule was capable of effective treatment against diffuse large B-cell lymphoma both in vitro and in vivo revealing efficient therapeutic capabilities with minimal toxic side effects; (3), metformin-derived CDs showed a unique nucleus targeting property, which suggests a huge potential for future nucleus-targeting drug delivery; (4), CDs have constantly shown the capability to inhibit the formation of amyloid precursor protein (APP), beta-amyloid $(A \beta)$ and $A \beta$ fibrils. $C D$ s are promising nanomedicine and drug nanocarriers to treat Alzheimer's disease (AD); (5) a pilot study showed a versatile nanocarrier could be assembled via the direct conjugation between distinct CDs to fulfill multitasks.
\end{abstract}

Keywords: carbon dots; drug nanocarrier; drug delivery system; blood-brain barrier penetration; lymphoma treatment; nucleus targeting drug delivery; alzheimer's disease treatment; nanoparticle assembly.

(C) 2020 by the authors. This article is an open-access article distributed under the terms and conditions of the Creative Commons Attribution (CC BY) license (https://creativecommons.org/licenses/by/4.0/).

\section{Funding}

This research was funded by the National Science Foundation, grant number 1809060 and 2041413.

\section{Acknowledgments}

This research has no acknowledgment.

\section{Conflicts of Interest}

The authors declare no conflict of interest. The funders had no role in the design of the study; in the collection, analyses, or interpretation of data; in the writing of the manuscript, or in the decision to publish the results. 